\title{
Restoring memory by optogenetic synchronization of hippocampal oscillations in an Alzheimer's disease mouse model
}

Eleonora Ambrad Giovannetti ${ }^{1}$, Stefanie Poll ${ }^{1}$, Daniel Justus ${ }^{2}$, Hiroshi Kaneko ${ }^{2}$, Falko Fuhrmann $^{2}$, Julia Steffen ${ }^{1}$, Stefan Remy ${ }^{2,3 *}$ and Martin Fuhrmann ${ }^{1 *}$

\footnotetext{
${ }^{1}$ Neuroimmunology and Imaging Group, German Center for Neurodegenerative Diseases (DZNE)

${ }^{2}$ Neuronal Networks Group, German Center for Neurodegenerative Diseases (DZNE), Bonn, Germany

${ }^{3}$ Department of Epileptology, University of Bonn Medical Center, Bonn, Germany
}

\section{*Corresponding authors:}

Martin Fuhrmann, Dr. rer. nat.

Neuroimmunology and Imaging Group

German Center for Neurodegenerative Diseases (DZNE)

Sigmund-Freud Str. 27

53127 Bonn

Germany

Email: martin.fuhrmann@dzne.de

Stefan Remy, Prof. Dr. med.

Neuronal Networks Group

German Center for Neurodegenerative Diseases (DZNE)

Sigmund-Freud Str. 27

53127 Bonn

Germany

Email: stefan.remy@dzne.de 
Disrupted neural oscillations are a feature of Alzheimer's disease (AD). We observed reduced frequency of theta oscillations in the hippocampal local field potential (LFP) in a mouse model of beta-amyloidosis. By restoring the temporal organization of theta oscillations using LFP-guided closed-loop optogenetic stimulation of parvalbuminpositive interneurons, we could rescue memory deficits of APP/PS1 mice in the novel object recognition test. Aberrant neuronal theta and gamma oscillations and neuronal hyperexcitability have been observed in patients and mouse models of Alzheimer's disease $(A D)^{1-8}$. Both pathophysiological phenomena may have detrimental effects on cognitive function. While aberrant oscillatory patterns are expected to interfere with mnemonic processing by disrupting the temporal coding within neuronal ensembles ${ }^{9}$, neuronal hyperexcitability is likely resulting in pathophysiological alterations of the neuronal input to output transformation $^{6,10}$. Novel interventional strategies should therefore act effectively at the network and the single cell level ${ }^{11}$. During mnemonic processing within the entorhinalhippocampal circuitry, theta oscillations in the $4-12 \mathrm{~Hz}$ frequency range are prominent in rodents and in humans ${ }^{9}$. They are driven by rhythmic activity of septo-hippocampal projections, which target hippocampal interneurons ${ }^{9}$. A reduced theta frequency has been observed in $A D$ mouse models and patients ${ }^{12-14}$. Notably, we and others have previously shown that $A D$-like pathology results in inhibitory interneuron dysfunction ${ }^{15}$. Specifically, parvalbumin positive $\left(\mathrm{PV}^{+}\right)$interneurons were critically affected in humans and mouse models of $A D^{7,16,17}$. Increasing their inhibitory function rescued spatial memory deficits ${ }^{7}$ and reduced $A \beta$-plaque load in the visual cortex ${ }^{3}$. Increasing neuronal inhibition was also beneficial in human $\mathrm{MCl}$ patients and mouse models following administration of levetiracetam, a pro-inhibitory antiepileptic drug ${ }^{18,19}$. Mechanistically, increasing inhibitory function of specific interneurons may represent a way to counteract both aberrant network activity patterns and increased cellular excitability. Therefore, we designed a closed-loop theta detection and optogenetic stimulation approach based on continuous monitoring of the hippocampal local field potential. This approach was targeted specifically at $\mathrm{PV}^{+}$interneurons and resulted in a simultaneous shift of theta frequency towards more physiological levels and an increase in hippocampal $\mathrm{PV}^{+}$mediated inhibition. We hypothesized that such an approach could be effective in improving memory in an APPswe/PS1 1 E9 (APP/PS1) mouse model of Alzheimer's disease - restoring the temporal organization of theta oscillations by shifting them to a frequency range that is observed under physiological conditions.

First, to quantify differences in hippocampal oscillations between wild type and APP/PS1 transgenic animals, we recorded CA1 local field potential in the bilateral str. radiata during open field exploration (Figure 1a, b). In the transgenic group, the peak theta frequency during locomotion was significantly decreased by $0.4 \mathrm{~Hz}$ compared to wild type animals (Figure 1c; $W T: 8.3 \pm 0.07 \mathrm{~Hz}, \mathrm{n}=24$ mice; APP/PS1: $7.9 \pm 0.07 \mathrm{~Hz}, \mathrm{n}=21$ mice). Neither the mean 
power of theta oscillations nor their mean duration was significantly altered between the two groups (Supplementary fig. 1). The mean running speed was significantly higher in transgenic animals (Figure 1d; $W T: 6.3 \pm 0.40 \mathrm{~cm} / \mathrm{sec}, \mathrm{n}=24$ mice; APP/PS1: $7.9 \pm 0.44$ $\mathrm{cm} / \mathrm{sec}$ ). Nevertheless, although theta frequency is well known to positively correlate with running speed ${ }^{20}$, the peak theta frequency was reduced. Travelled distance and time spent in the center of the arena were not different between the groups (Figure 1e, f).

Next, we tested the effect of optogenetic stimulation of $\mathrm{PV}^{+}$interneurons on the hippocampal LFP. Therefore, we bilaterally injected rAAV2-Ef1a-DIO C1V1 (t/t)-TS-mCherry into the CA1 subregions of PV-Cre mice on wild type or APP/PS1 genetic background, thereby restricting the expression of $\mathrm{C} 1 \mathrm{~V} 1$ to $\mathrm{PV}^{+}$interneurons (Figure 2a, b). As controls, an additional group of mice with the identical genetic background was injected with a construct encoding a loxPflanked fluorescent reporter protein ( $r A A V 1-C A G-F L E X$-tdTomato). Bilateral optical fibers were implanted, terminating above the transfected region (Figure 2a, b; Supplementary figure 5). Light stimulation significantly increased the power of the hippocampal oscillations at the frequency bands at which stimulation was performed in C1V1 injected animals, but not in the Sham injected group (Figure. $2 \mathrm{c}$ and $2 \mathrm{~d}$; Sham: $\mathrm{n}=4$ to 5 animals; C1V1: $\mathrm{n}=6$ animals; Supplementary figure 4).

There are two plausible and mutually non-exclusive mechanisms by which optogenetic stimulation of $\mathrm{PV}^{+}$interneurons could lead to an improvement of memory performance in APP/PS1 mice: First, the stimulation of $\mathrm{PV}^{+}$interneurons in the theta range is expected to lead to a rhythmic recruitment of these interneurons. As demonstrated before, such a rhythmic, synchronous firing of many $\mathrm{PV}^{+}$interneurons will likely result in a stimulationfrequency dependent field potential oscillation ${ }^{20,21}$. It is possible that by (i) shifting the mean theta frequency towards more physiological levels in APP/PS1 mice and by (ii) increasing the rhythmicity of the oscillation, the temporal coding of memory-relevant information would be improved. The relative timing of synaptic input and action potential firing in relation to the phase of the theta oscillation has been implicated to be relevant for the encoding and retrieval of memories (for ref. $\mathrm{see}^{22,23}$ ). Thus, by defining the time window at which inhibition permits synaptic input to cause action potential firing, the effectiveness of encoding could be enhanced. Second, neuronal hyperexcitability in AD models has been shown to profoundly alter the input-output conversion of neurons resulting in higher firing rates and a shift of the output pattern towards more burst firing ${ }^{6,10}$. Since the synchronous and repetitive stimulation of interneurons is expected to result in a sustained increase in inhibition during the stimulation periods, it is expected to cause a shift of the input-output function in hippocampal neurons towards a reduced spike output at a given input strength ${ }^{24}$. We therefore designed an experimental approach that aimed at distinguishing the effects of increased rhythmicity of pyramidal cell output on memory performance from effects caused by a general increase in 
inhibitory strength. Our approach aimed at maintaining an equal inhibitory strength in two dissimilar stimulation patterns: Both stimulation patterns consisted of equal numbers of stimuli, but differed in the temporal precision at which pulses were delivered. In the "rhythmic" stimulation protocol the interpulse interval was kept stable so that the light pulses were equally spaced. The "arrhythmic stimulation" protocol introduced a random jitter in the interpulse interval duration that did not exceed $30 \mathrm{~ms}$. Before applying these protocols in a closed-loop optogenetic feedback stimulation in vivo, we carried out whole-cell patch-clamp recordings of $\mathrm{PV}^{+}$and $\mathrm{CA} 1$ pyramidal neurons (PYR) in acute slices of wild type PV-Cre mice injected with rAAV2-Ef1a-DIO C1V1 (t/t)-TS-mCherry (Figure 3a). These experiments confirmed a) the high reliability of light-induced action potential generation in $\mathrm{PV}+$ interneurons, b) an inhibitory effect of $\mathrm{PV}^{+}$stimulation on the membrane potential of CA1 pyramidal neurons and most importantly c) no difference in the strength of inhibition evoked by both stimulation protocols (Figure $3 b-d$ ). The latter was determined by a comparison of IPSC amplitudes evoked by repetitive stimulation. Subsequently, these protocols were implemented into closed-loop optogenetic feedback stimulation paradigms; two groups of mice were subjected to either the "rhythmic" or the "arrhythmic" feedback stimulation during a novel object recognition test (NOR). In both cases, the optogenetic feedback stimulation was driven by the endogenous theta oscillations recorded in the LFP of the behaving mice. In particular, an increase in the theta oscillation power would trigger the optogenetic stimulation at a frequency equal to the one detected in the LFP, plus an additional $0.5 \mathrm{~Hz}$ to compensate for the deficit measured in APP/PS1 transgenic mice (Figure 3e). The NOR was carried out $24 \mathrm{~h}$ after the memory acquisition phase (Figure $3 \mathrm{f}$ ), and discrimination indices (DI) were calculated to describe the degree of memory retention (See Methods). The rhythmic stimulation protocol resulted in an improved recognition memory in C1V1-injected PVCre::APP/PS1 mice in contrast to Sham-injected PV-Cre::APP/PS1 mice (Figure 3g; APP/PS1-Sham: $\mathrm{DI}=41.8 \% \pm 8.18 \% ; \mathrm{n}=11$ mice; APP/PS1-C1V1: $\mathrm{DI}=66.6 \pm 7.46 \%, \mathrm{n}=$ 11 mice). Interestingly, the rhythmic optogenetic stimulation of $\mathrm{PV}^{+}$interneurons in the C1V1injected wild type group impaired recognition memory compared to Sham-injected controls (Figure 3g; WT-Sham: $\mathrm{DI}=73.2 \% \pm 7.03 \%, \mathrm{n}=8, W T-C 1 V 1: \mathrm{DI}=39.8 \% \pm 6.18 \%, \mathrm{n}=11$ ). Notably, while the rhythmic optogenetic stimulation could rescue impaired recognition memory, the arrhythmic stimulation protocol was not sufficient to improve novel object discrimination of C1V1-injected PV-Cre::APP/PS1 mice compared to Sham-injected PVCre::APP/PS1 mice (Fig. 3h; APP/PS1-Sham: DI = 46.3\% $\pm 14.3, \mathrm{n}=7 ;$ APP/PS1-C1V1: DI $=49.4 \% \pm 13.4 \%, n=5$ ). Mean travelled distance was comparable between all experimental groups, indicating no effect of the light stimulation on locomotor activity (Supplementary figure 2). Light delivery per se had no effect on the LFP of Sham-injected mice subjected to 
the rhythmic feedback stimulation (Supplementary figure $3 \mathrm{a}-\mathrm{g}$ ), nor on the LFP of C1V1 injected mice subject to the arrhythmic feedback stimulation (Supplementary figure $3 \mathrm{~h}-\mathrm{k}$ ).

Taken together, we found that the peak frequency of theta oscillations in APP/PS1 mice was significantly reduced when compared to wild type littermates (albeit their higher movement speeds), a finding which is consistent with previous reports in patients and $A D$ mouse models $^{12-14}$. Most remarkably, the closed-loop rhythmic optogenetic stimulation of hippocampal $\mathrm{PV}^{+}$interneurons effectively rescued the impaired recognition memory of APP/PS1 mice during the novel object recognition task bringing it back to levels comparable to wild type mice. This rescue was not observed upon arrhythmic stimulation, suggesting that memory processes may benefit from an optogenetic enhancement of theta rhythmicity and an associated improvement in temporal coding rather than from an increase in bulk inhibition alone. The result that optogenetic treatment of wild type mice had detrimental effects on memory performance underscores that, physiologically, the temporal coding of neuronal ensemble activation is optimally tuned, in contrast to pathophysiological conditions. Thus, it is possible that shifting the frequency of the endogenous theta rhythm towards higher frequencies and increasing bulk inhibition brought this optimized tuning in disarray, while the same procedure effectively restored the prevalent temporal asynchrony and hyperexcitability of the hippocampal circuitry, favoring more effective memory processing in APP/PS1 mice.

In conclusion, we showed that restoring the disrupted theta rhythmicity measured in PVCre::APP/PS1 transgenic mice by means of rhythmic optogenetic $\mathrm{PV}^{+}$interneuron stimulation rescued long-term memory deficits in a novel object recognition task. Electrical deep brain stimulation has been used in several brain regions in patients and positive effects on memory performance have been reported in studies in humans including AD patients ${ }^{25}$. However, it is likely that the performance enhancing effect of deep brain stimulation is multi-factorial and mechanistically not precisely defined ${ }^{25,26}$. Our results using optogenetic stimulation now identify $\mathrm{PV}^{+}$interneurons as a target and a mean to restore neural synchronicity. Ultimately, this may serve to guide the development of more effective and specific interventional stimulation strategies in humans. 


\section{Acknowledgements}

This work was supported by the DZNE, grants from the Deutsche Forschungsgemeinschaft (SFB1089 B01, C01, B06, C06 to M.F. and S.R.), ERA-NET MicroSynDep, and the CoEN initiative (3018).

\section{Author contributions}

E.A.G. performed in vivo experiments, analyzed the data and prepared figures. H.K. performed the patch-clamp recordings with optogenetic stimulation in acute brain slices and analyzed the data. S.P. and F.F. provided assistance for surgical procedures. J.S. provided technical assistance with perfusions and histology. D.J. and S.R. designed the closed-loop stimulation, D.J. wrote the custom IgorPro scripts for closed-loop detection and stimulation, and the MATLAB scripts for field potential analysis. S.R, M.F. and E.A.G. wrote the manuscript, with contributions from all co-authors. S.R. and M.F. coordinated research. M.F. supervised the research project and designed the experiments. 


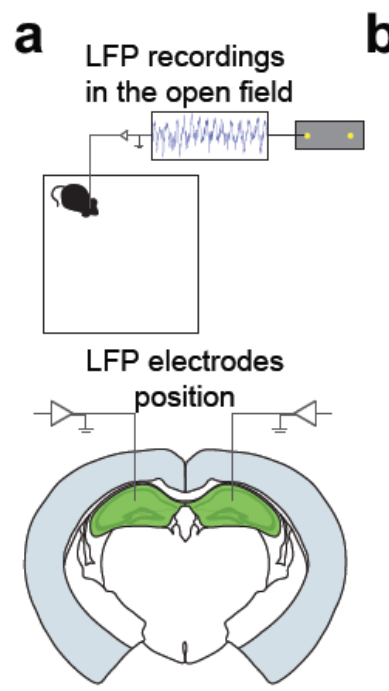

b
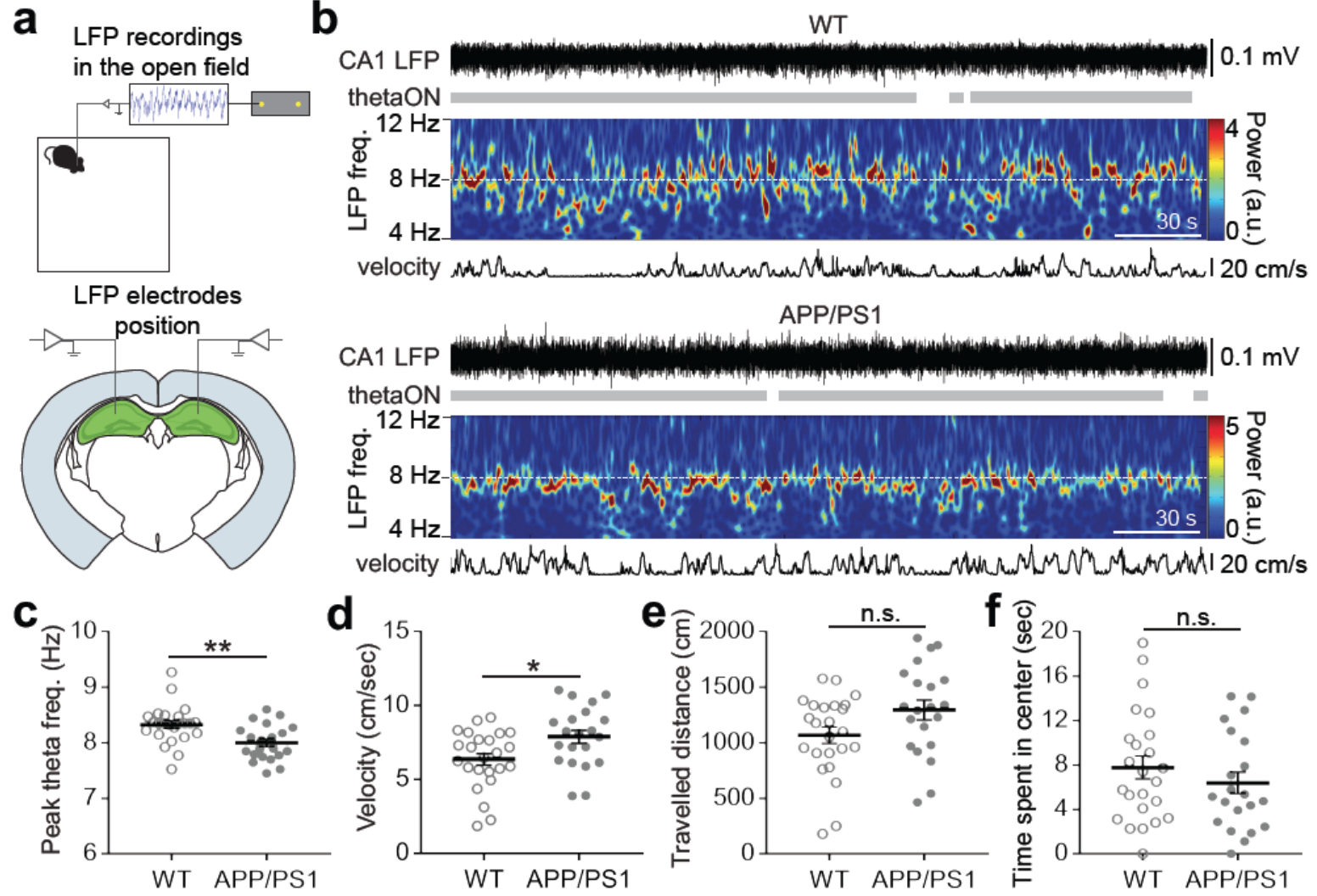

Figure 1. Slower theta oscillations in aged APP/PS1 mice.

(a) Schematics of LFP recording configuration. (b) Exemplary spectrograms showing the slower peak theta frequency recorded in APP/PS1 compared to wild type (WT) mice during open field exploration. Theta ON refers to the intervals of increased theta power detected during offline analyses. (c) Peak theta frequency of WT and APP/PS1 mice, detected during ThetaON intervals $\left({ }^{* *} p<0.01\right.$; unpaired Student's t-test). (d) Mean velocity during epochs of locomotion (point velocity $>0.5 \mathrm{~cm} / \mathrm{sec}$ ); ${ }^{*} p<0.05$, unpaired Student's t-test. (e) Total travelled distance. (f) Time spent in the center of the arena. For $\mathbf{e}$ and $\mathbf{f}, \mathrm{n} . \mathrm{s} .=p>0.05$, not significant; unpaired Student's t-test. For $\mathbf{c}$ to $\mathbf{f}, \mathrm{WT}: \mathrm{n}=24$, APP/PS1: $n=21$. Dots represent individual mice, bars show mean \pm SEM. Individual mouse values result from the average of 2-6 recordings of 3 min each. 
a

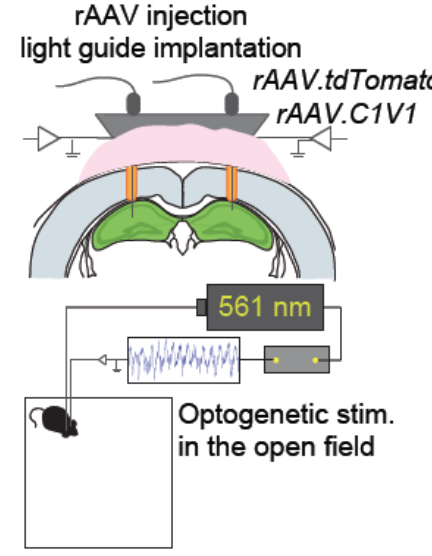

b

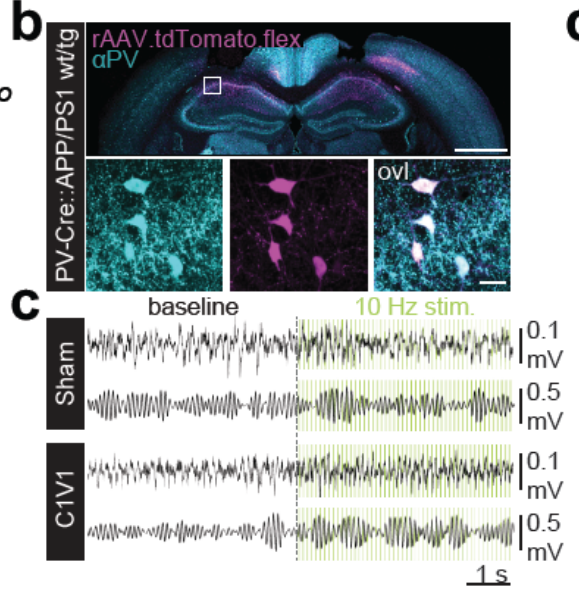

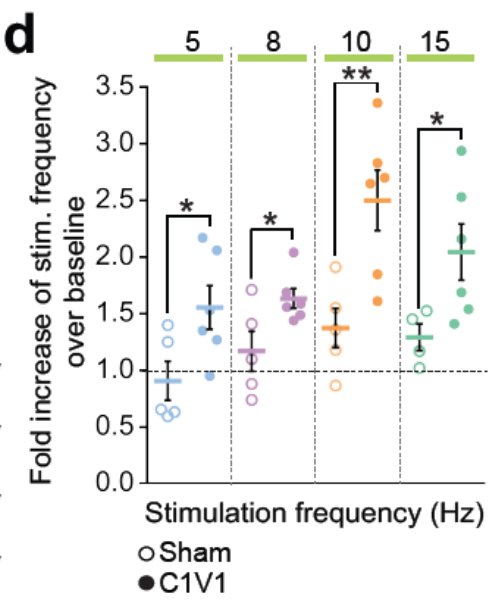

Figure 2. Optogenetic stimulation of $\mathrm{PV}^{+}$interneurons drives LFP frequencies.

(a) Schematics of experimental setup. (b) Exemplary picture of rAAV expression in $\mathrm{PV}^{+}$ interneurons in the hippocampus. Scale bars: $1 \mathrm{~mm}$ (upper panel) and $20 \mu \mathrm{m}$ (lower panel), ovl = overlap (c) Exemplary LFP traces recorded from mice injected with rAAV-tdTomato (Sham) and rAAV-C1V1 (C1V1) during a $10 \mathrm{~Hz}$ light $(561 \mathrm{~nm})$ stimulation protocol. Upper traces: filtered LFP, 3-200 Hz. Lower traces: filtered LFP, 8-12 Hz. (d) Fold increase of stimulation frequency over baseline (Sham: $n=5$ mice for all frequencies but $15 \mathrm{~Hz}$, where $n=4$, C1V1: $n=6$ mice for all frequencies; ${ }^{*} p<0.05,{ }^{* *} p<0.01$; unpaired Student's t-tests). The dashed line represents the baseline to which the fold increase was normalized. Dots represent individual mouse values, bars show mean \pm SEM. Individual mouse values result from the average of 4-6 stimulation protocols. 
a

a In vitro recording + optogenetic stim.



e

In vivo feedback-loop optogenetic stimulation stimulation@ recorded theta $+0.5 \mathrm{~Hz}$

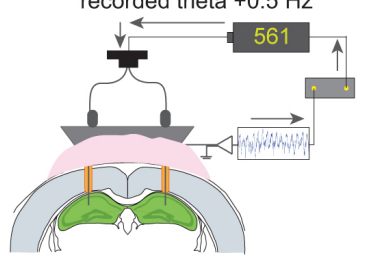

b

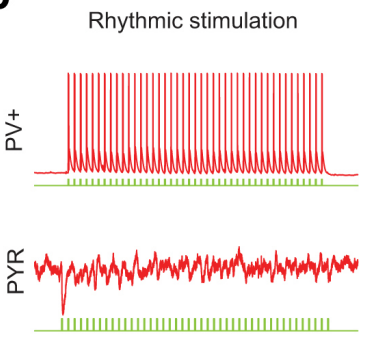

f

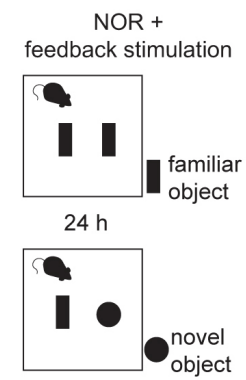

C

Arrhythmic stimulation

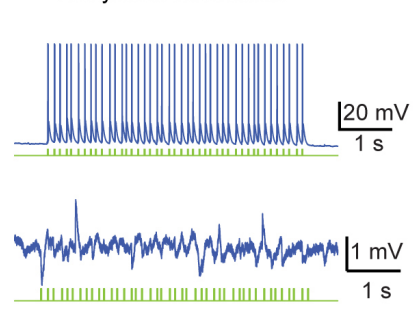

g Rhythmic stimulation



d

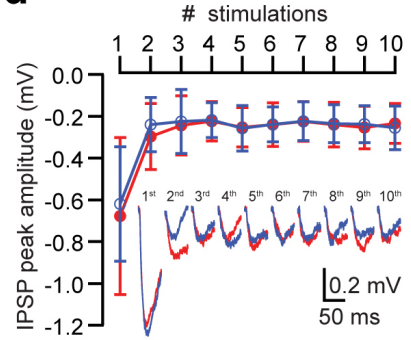

h

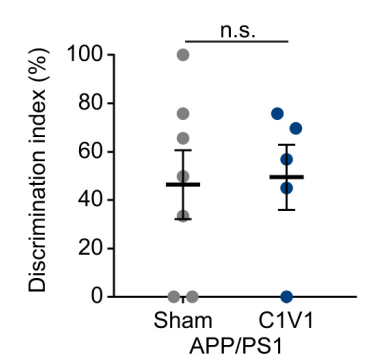

Figure 3. Enhanced neural synchronization via $\mathrm{PV}^{+}$interneuron stimulation improves memory.

(a) Schematics of the in vitro recording and optogenetic stimulation configuration. (b, c) Exemplary recordings from a C1V1-expressing $\mathrm{PV}+$ interneuron and a CA1 pyramidal neuron (PYR) during rhythmic (b) and arrhythmic (c) $8.5 \mathrm{~Hz}$ light stimulation. (d) Average peak IPSP amplitudes recorded from CA1 pyramidal neurons plotted against the number of optogenetic stimulations comparing rhythmic (red) and arrhythmic (blue) stimulation of C1V1expressing PV+ interneurons. Inset: individual example. (e) Schematics illustrating the in vivo recording and feedback optogenetic stimulation configuration. (f) Schematics of the novel object recognition (NOR) test with a $24 \mathrm{~h}$ interval time. Optogenetic feedback stimulation was carried out upon theta frequency detection during the NOR test. $(\mathbf{g}, \mathbf{h})$ Discrimination index of wild type (WT) and APP/PS1 mice expressing either C1V1 or tdTomato (Sham) in $\mathrm{PV}^{+}$ interneurons. Mice performed the NOR task during rhythmic $(\mathrm{g})$ or arrhythmic $(\mathrm{h})$ feedbackloop optogenetic stimulation of PV+ interneurons; * $p<0.05$, two-way ANOVA with HolmSidak's correction (Interaction factor $=29.3 \%$ of total variation, ${ }^{* * *} p<0.001$; row and column factors $=0.18 \%$ and $0.64 \%$ of total variation, $p>0.05$ ); n.s. $=p>0.05$, not significant; unpaired Student's t-test. (For g, WT-Sham: $n=8$, APP/PS1-Sham: $n=11$, WT-C1V1: $n=11$, APP/PS1-C1V1: $n=11$. For $\mathbf{h}$, APP/PS1-Sham: $n=7$, APP/PS1-C1V1: $n=5$. Dots represent individual mouse values, bars show mean \pm SEM). 


\section{References}

1. Busche, M.A., et al. Critical role of soluble amyloid-beta for early hippocampal hyperactivity in a mouse model of Alzheimer's disease. Proc Natl Acad Sci U S A 109, 87408745 (2012).

2. Fu, H., et al. Tau Pathology Induces Excitatory Neuron Loss, Grid Cell Dysfunction, and Spatial Memory Deficits Reminiscent of Early Alzheimer's Disease. Neuron 93, 533-541 e535 (2017).

3. laccarino, H.F., et al. Gamma frequency entrainment attenuates amyloid load and modifies microglia. Nature 540, 230-235 (2016).

4. Palop, J.J., et al. Aberrant excitatory neuronal activity and compensatory remodeling of inhibitory hippocampal circuits in mouse models of Alzheimer's disease. Neuron 55, 697711 (2007).

5. Scott, L., et al. Age-dependent disruption in hippocampal theta oscillation in amyloidbeta overproducing transgenic mice. Neurobiology of aging 33, 1481 e1413-1423 (2012).

6. Siskova, Z., et al. Dendritic structural degeneration is functionally linked to cellular hyperexcitability in a mouse model of Alzheimer's disease. Neuron 84, 1023-1033 (2014).

7. Verret, L., et al. Inhibitory interneuron deficit links altered network activity and cognitive dysfunction in Alzheimer model. Cell 149, 708-721 (2012).

8. Vossel, K.A., et al. Incidence and impact of subclinical epileptiform activity in Alzheimer's disease. Annals of neurology 80, 858-870 (2016).

9. Buzsaki, G. Theta oscillations in the hippocampus. Neuron 33, 325-340 (2002).

10. Hall, A.M., et al. Tau-dependent Kv4.2 depletion and dendritic hyperexcitability in a mouse model of Alzheimer's disease. J Neurosci 35, 6221-6230 (2015).

11. Canter, R.G., Penney, J. \& Tsai, L.H. The road to restoring neural circuits for the treatment of Alzheimer's disease. Nature 539, 187-196 (2016).

12. Goutagny, R., et al. Alterations in hippocampal network oscillations and theta-gamma coupling arise before Abeta overproduction in a mouse model of Alzheimer's disease. Eur $J$ Neurosci 37, 1896-1902 (2013).

13. Cayzac, S., et al. Altered hippocampal information coding and network synchrony in APP-PS1 mice. Neurobiology of aging 36, 3200-3213 (2015).

14. Jelic, V., et al. Quantitative electroencephalography in mild cognitive impairment: longitudinal changes and possible prediction of Alzheimer's disease. Neurobiology of aging 21, 533-540 (2000).

15. Schmid, L.C., et al. Dysfunction of Somatostatin-Positive Interneurons Associated with Memory Deficits in an Alzheimer's Disease Model. Neuron 92, 114-125 (2016).

16. Takahashi, $H_{\text {. }}$, et al. Hippocampal interneuron loss in an APP/PS1 double mutant mouse and in Alzheimer's disease. Brain structure \& function 214, 145-160 (2010).

17. Satoh, J., Tabira, T., Sano, M., Nakayama, H. \& Tateishi, J. Parvalbuminimmunoreactive neurons in the human central nervous system are decreased in Alzheimer's disease. Acta neuropathologica 81, 388-395 (1991).

18. Sanchez, P.E., et al. Levetiracetam suppresses neuronal network dysfunction and reverses synaptic and cognitive deficits in an Alzheimer's disease model. Proc Natl Acad Sci U S A 109, E2895-2903 (2012).

19. Bakker, A., et al. Reduction of hippocampal hyperactivity improves cognition in amnestic mild cognitive impairment. Neuron 74, 467-474 (2012).

20. Fuhrmann, F., et al. Locomotion, Theta Oscillations, and the Speed-Correlated Firing of Hippocampal Neurons Are Controlled by a Medial Septal Glutamatergic Circuit. Neuron 86, 1253-1264 (2015).

21. Bender, F., et al. Theta oscillations regulate the speed of locomotion via a hippocampus to lateral septum pathway. Nat Commun 6, 8521 (2015).

22. Hasselmo, M.E. \& Stern, C.E. Theta rhythm and the encoding and retrieval of space and time. Neuroimage 85 Pt 2, 656-666 (2014).

23. Korotkova, T., et al. Reconciling the different faces of hippocampal theta: The role of theta oscillations in cognitive, emotional and innate behaviors. Neuroscience and biobehavioral reviews 85, 65-80 (2018). 
24. Isaacson, J.S. \& Scanziani, M. How inhibition shapes cortical activity. Neuron $\mathbf{7 2}$, 231-243 (2011).

25. Hardenacke, K., et al. Deep brain stimulation as a tool for improving cognitive functioning in Alzheimer's dementia: a systematic review. Front Psychiatry 4, 159 (2013).

26. Kuhn, J., Buhrle, C.P., Lenartz, D. \& Sturm, V. Deep brain stimulation in addiction due to psychoactive substance use. Handb Clin Neurol 116, 259-269 (2013).

27. Oostenveld, R., Fries, P., Maris, E. \& Schoffelen, J.M. FieldTrip: Open source software for advanced analysis of MEG, EEG, and invasive electrophysiological data. Computational intelligence and neuroscience 2011, 156869 (2011). 

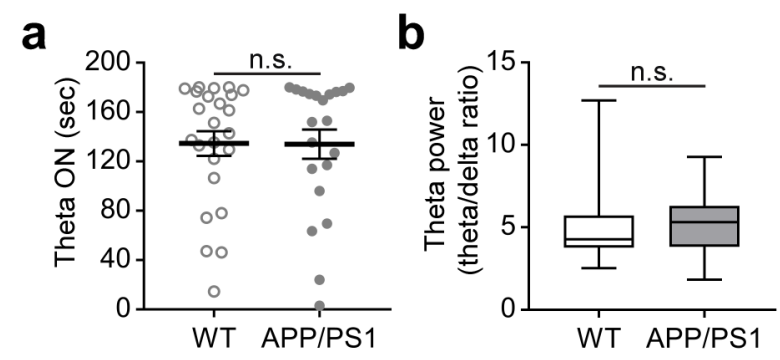

Supplementary figure 1. Duration and power of theta oscillations were not altered in APP/PS1 transgenic mice. (a) Average duration of theta oscillations during active exploration in the open field (2-6 recordings of $3 \mathrm{~min}$ per mouse); unpaired Student's t-test. The graph represents individual mouse values, mean \pm SEM. (b) Amplitude of the recorded theta oscillations defined as the ratio of theta band $(5-12 \mathrm{~Hz})$ over the delta band $(4-5 \mathrm{~Hz})$; Mann-Whitney test. Whisker plots represent median, $25^{\text {th }}$ and $75^{\text {th }}$ percentiles. For $\mathbf{a}$ and $\mathbf{b}$ WT: n=24, APP/PS1: n=21; n.s.= not significant. 

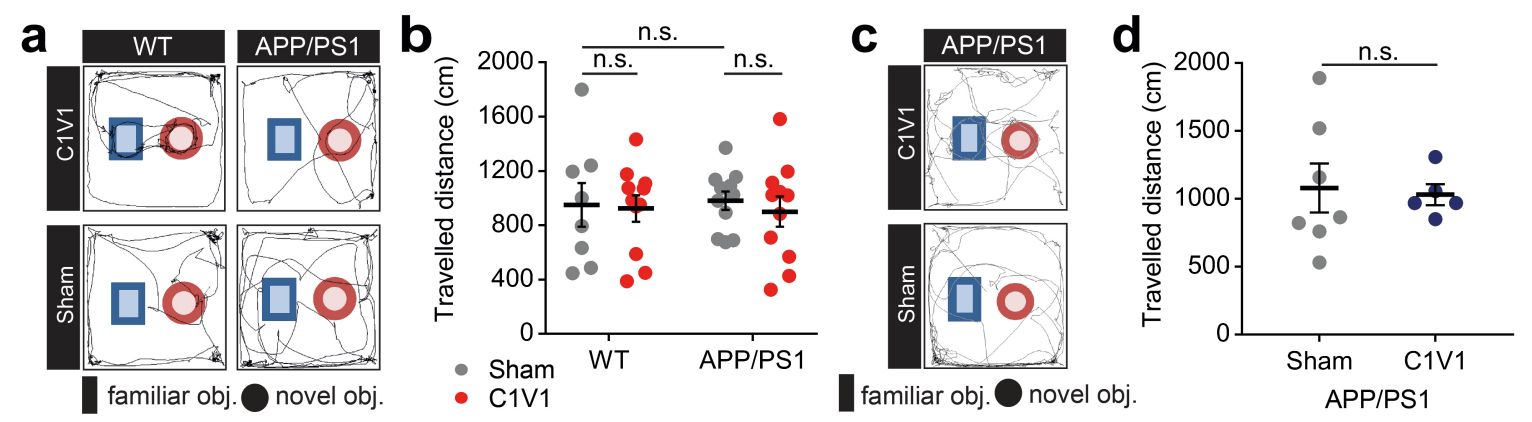

Supplementary figure 2. Travelled distance during the NOR test was unaltered upon feedback loop stimulation and unchanged between genotypes. (a) Rhythmic stimulation: Exemplary traces of single mice exploring novel (red circle) or familiar (blue rectangle) object in the open field. (b) Total travelled distance during the test; n.s.= not significant, two-way ANOVA with Holm-Sidak's correction. (c) Arrhythmic stimulation: Exemplary traces of single mice exploring novel (red circle) or familiar (blue rectangle) object in the open field. (d) Total travelled distance during the test; $n . s .=p>0.05$, not significant; unpaired Student's t-test. Dots represent individual mouse values, bars show mean \pm SEM. 


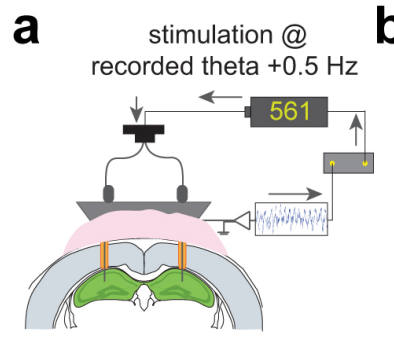

Rhythmic stimulation

C WT-C1V1

$\theta$ detection stimulation

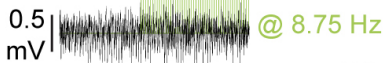

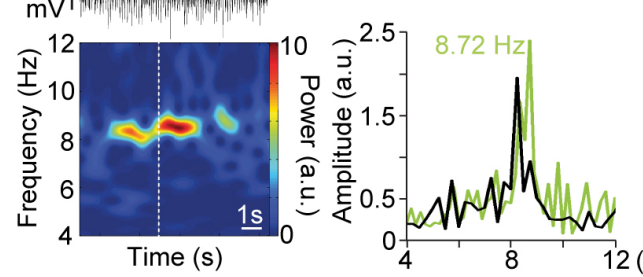

WT-Sham

$\theta$ detection stimulation

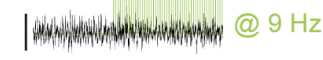

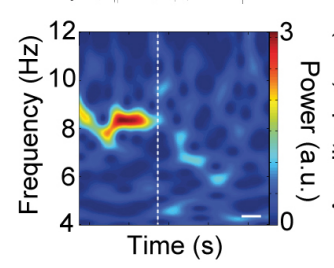

e

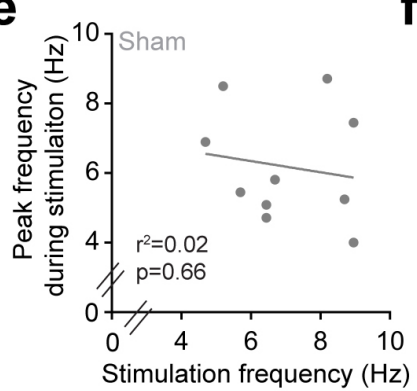

Arrhythmic stimulation

h APP/PS1-C1V1

$\theta$ detection stimulation

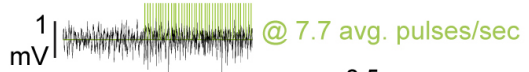
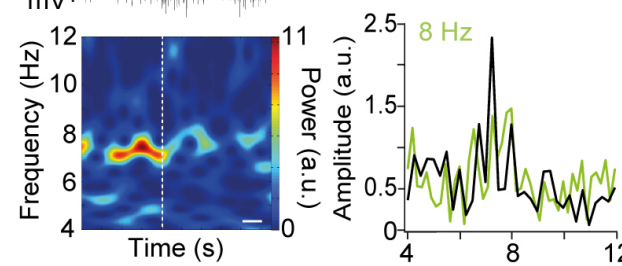

d APP/PS1-C1V1

$\theta$ detection stimulation

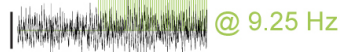
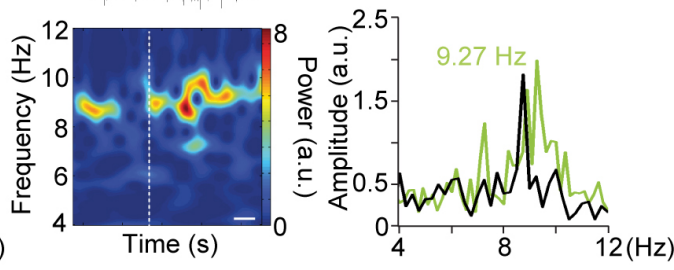

APP/PS1-Sham

$\theta$ detection stimulation

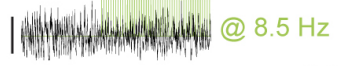
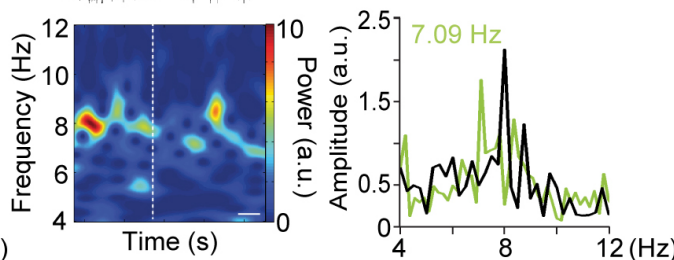

$\mathbf{g}$

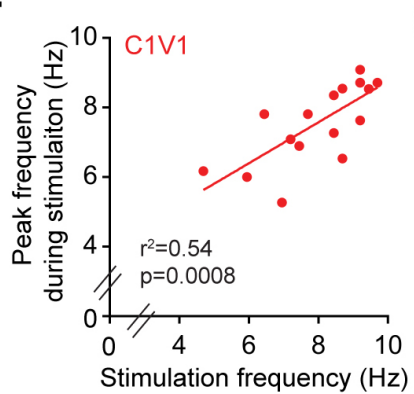

i

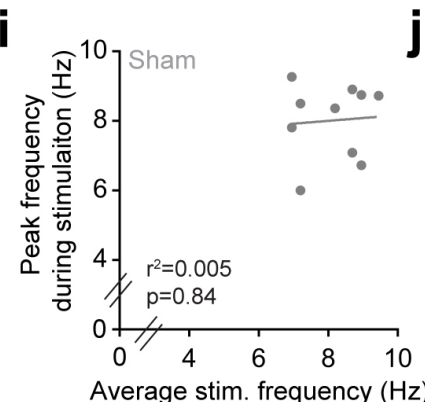

Average stim. frequency $(\mathrm{Hz})$



APP/PS1-Sham

$\theta$ detection stimulation

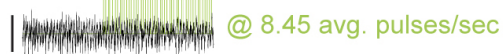
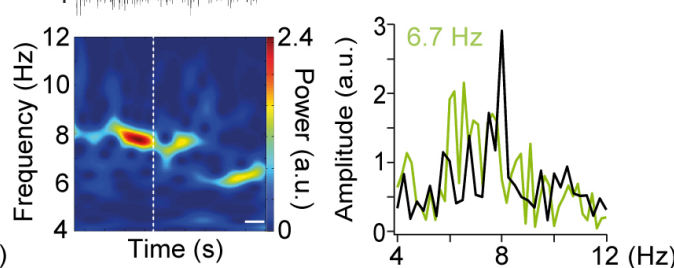

k

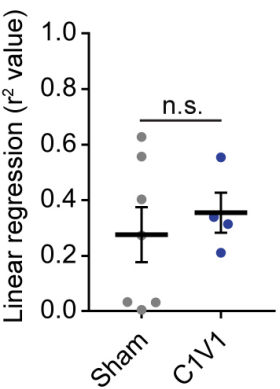


Supplementary figure 3 . Effect of the closed-loop feedback stimulation of $\mathrm{PV}^{+}$ interneurons on the hippocampal LFPs.. (a) Schematics of the closed feedback-loop optogenetic stimulation. (b) Exemplary LFP trace and respective spectrogram showing the activation of the closed loop throughout one recording. (c-d) Rhythmic stimulation: Exemplary LFP epoch, respective spectrograms and fast Fourier transform of a single stimulation event driven by the endogenous theta frequency detected in the LFP of C1V1(upper panel) and Sham-injected (lower panel) WT mice (c). Respective examples for APP/PS1 mice (d). Scale bars: $0.5 \mathrm{mV}$ and $1 \mathrm{sec}$ for all spectrograms. Note that in Shaminjected mice (WT-Sham) the stimulation has no effect on the LFP. (e-f) Exemplary $x-y$ plot showing the stimulation frequency on the (x-axis) and the respective peak frequency (y-axis) recorded during stimulation epochs for a Sham- (e) and a C1V1-(f) injected mouse. (g) Cumulative $r^{2}$ value for Sham- and C1V1-injected animals (Sham: $n=16$; C1V1: $n=20$ mice; ${ }^{*} \mathrm{p}<0.05$, unpaired Student's t-test). (h) Arrhythmic stimulation: Exemplary LFP epoch, respective spectrograms and fast Fourier transform of a single stimulation event driven by the endogenous theta frequency detected in the LFP of APP/PS1 mice. Scale bars: $1 \mathrm{mV}$ and $1 \mathrm{sec}$ for all spectrograms. (i-j) Exemplary $x-y$ plot showing the average stimulation frequency on the ( $x$ axis) and the respective peak frequency ( $y$ axis) recorded during arrhythmic stimulation epochs for a Sham (i) and a C1V1 (j) injected mouse. (k) Cumulative $r^{2}$ value for Sham- and C1V1-injected animals (Sham: $n=7 ; C 1 V 1: n=4$ mice; $n . s .=p>0.05$, not significant, unpaired Student's t-test). For e-f and $\mathbf{i}-\mathbf{j}$ : each dot represents a single stimulation event. For $\mathbf{g}$ and $\mathbf{k}$ : dots represent individual mouse values, bars show means \pm SEM. 


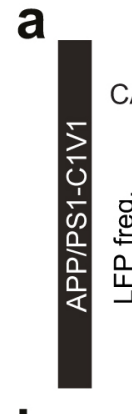

b

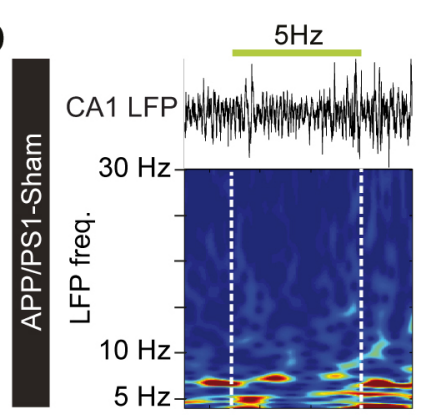

C

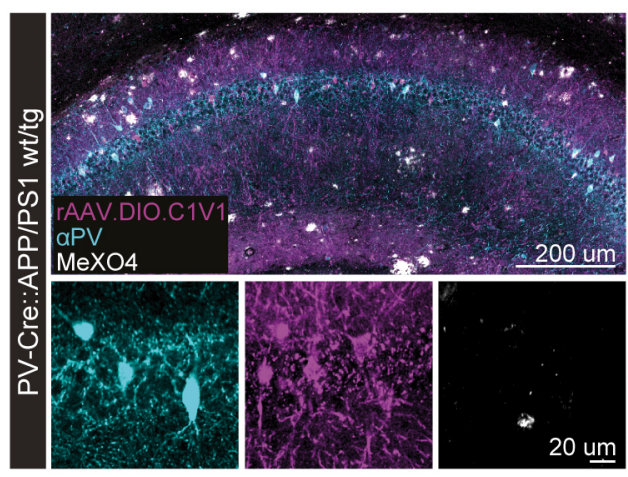

$8 \mathrm{~Hz}$



$8 \mathrm{~Hz}$
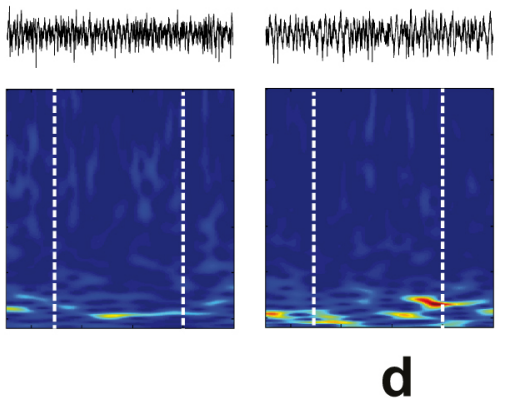

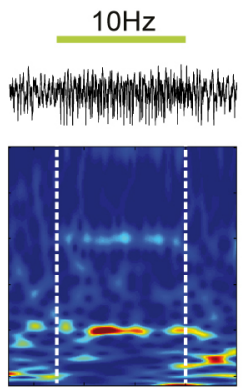

$10 \mathrm{~Hz}$

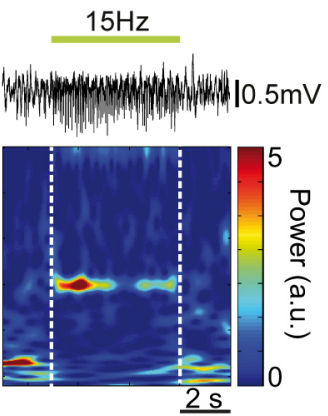

$15 \mathrm{~Hz}$

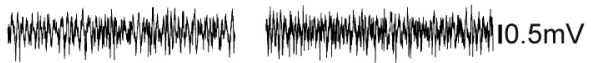
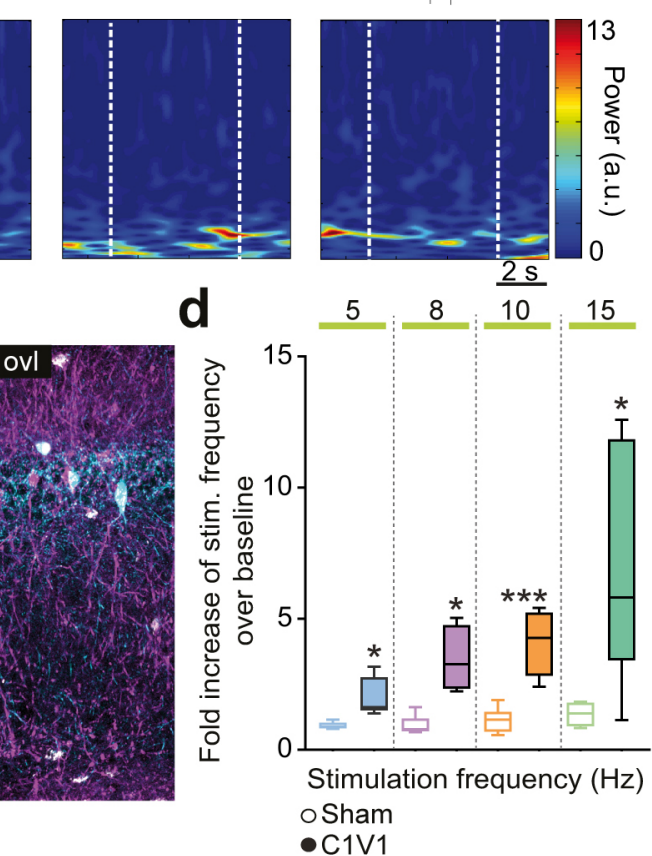

Supplementary figure 4. Optogenetic stimulation of $\mathrm{PV}^{+}$interneurons drives LFP frequencies. This data was acquired in the mice employed for the arrhythmic stimulation (figure 3 e to $\mathbf{h}$ ). (a, b) Exemplary spectrograms showing the effect of $5,8,10$ and $15 \mathrm{~Hz}$ laser stimulation of the LFP of a C1V1-(a) and a Sham-injected (b) PV-Cre::APP/PS1 mouse. Each spectrogram corresponds to a single stimulation epoch. (c) Exemplary immunofluorescent staining showing colocalization of the rAAV.DIO.C1V1 with the aPV antibody. $A \beta$ plaques are stained by the MeXO4 dye. (c) Fold increase of stimulation frequency over baseline. Sham: $n=5-6$ mice per frequency; C1V1: $n=4-5$ mice per frequency. ${ }^{*} p<0.05$, unpaired Student's t-tests with Welch's correction; ${ }^{* *} p<0.01$, unpaired Student's t-test. The dashed line represents the baseline to which the fold increase was normalized. Whisker plots represent median, $25^{\text {th }}$ and $75^{\text {th }}$ percentiles. Individual mouse values result from the average of 2-6 stimulation protocols. 



Supplementary figure 5. Examples of electrode and light fiber placement. Brightfield confocal images showing position of the electrode tip in the stratum radiatum of the hippocampal CA1 area of 9 exemplary mice (as indicated by the arrowheads). Only one of the two hemispheres is shown. Scale bars: $1 \mathrm{~mm}$ in all panels. 


\section{Materials and methods}

\section{Animals}

Experiments were performed on male and female PV-Cre::APP/PS1 mice. These were generated by crossbreeding B6.129P2-Pvalb ${ }^{\mathrm{tm} 1(\mathrm{cre}) \text { Arbr }} / \mathrm{J}$ mice (named PV-Cre, Stock number: 008069, The Jacksons Laboratory) to B6.Cg-Tg(APPswe,PSEN1dE9)85Dbo/Mmjax mice (named APP/PS1, MMRRC stock number 034832, The Jacksons Laboratory). Mice were housed with their littermates under specific pathogen free conditions and were given food and water ad libitum. All experiments were conducted during the light phase of the $12 \mathrm{~h}$ dark/light cycle (lights on at 6:00 am, lights off at 6:00 pm) in the same room, lit by a normal neon lamp. The experimental procedures were in accordance with guidelines established by the DZNE and were approved by the government of North-Rhine-Westphalia. At the end of the experimental procedures, wild type mice were aged $14 \pm 3.1$ months and transgenic mice $15 \pm 3.5$ months (mean $\pm S D$ ) for experiments shown in Figures 1, 2 and $3 \mathrm{e}-\mathrm{h}$. For the second cohort of mice, we employed only APP/PS1 transgenic animals (Figure $3 \mathrm{~h}$ ), and average age at the end of experimental procedures was $14 \pm 2.2$ months (mean $\pm S D$ ).

\section{Surgical procedures}

\section{Stereotactic viral injections}

Mice were anesthetized with a mixture of ketamine/xylazine $(0.13 / 0.01 \mathrm{mg} / \mathrm{g}$ body weight; Bela Pharm GmbH\&Co. KG, Vechta and Rompun 2\%, Bayer Vital GmbH, Leverkusen, DE) injected intraperitoneally. Additionally, mice received temgesic as analgesic $(0.05 \mathrm{mg} / \mathrm{kg}$; RB Pharmaceuticals Limited, Mumbai, IN), and dexamethasone as anti-inflammatory drug $(0.2$ $\mathrm{mg} / \mathrm{kg}$; Sigma Aldrich, Taufkirchen, DE), all injected subcutaneously prior to the surgery. A layer of eye-ointment (Bepanthen, Bayer Vital $\mathrm{GmbH}$, Leverkusen) was applied on the eyes of the mice to prevent de-hydration. Upon absence of toe-pinch reflex, a small skin incision was made to expose the skull. Two small holes ( $\sim 0.5 \mathrm{~mm}$ diameter) were opened through the skull using a dental drill. AAV2-Ef1a-DIO C1V1 (t/t)-TS-mCherry and AAV1-CAG-FLEXtdTomato were bilaterally injected in the hippocampi of different groups of wild type and transgenic PV-Cre:APP/PS1 mice. $1 \mu \mathrm{l}$ of virus was injected in each hemisphere at a speed of $100 \mathrm{nl} / \mathrm{min}$, above the CA1 area (AP: $-1.8 \mathrm{~mm}$ from bregma, ML: $+-1.5 \mathrm{~mm}$ from midline and DV: $+1.1 \mathrm{~mm}$ from brain surface). Injections were performed using a Hamilton syringe with a 34G needle (World Precision Instruments, Berlin, DE). Injection volume and speed were regulated using an UltraMicroPump (World Precision Instruments, Berlin, DE), and a micromanipulator (Luigs and Neumann, Ratingen, DE) was employed for stereotactic motion control. The needle was kept in the brain for 10 minutes prior to retraction. Finally, the skin was sutured with surgical wire (Vicryl, Ethicon, Norderstedt, DE). Mice were provided with analgesic $(0.05 \mathrm{mg} / \mathrm{kg})$ during three consecutive days following the surgery. Double fiber optic cannulas (light guides) and electrodes were implanted earliest 1 week and latest 3 weeks after stereotactic AAV-injections.

\section{Optic ferrules and LFP electrodes implantation}

Mice were anesthetized and provided with analgesic, antibiotic and anti-inflammatory as described above. Double fiber optic cannulas (B284-3015-2.5, TFC_300/370 - 0.22-2.5 mm, TSM3_FLT, Doric lenses, Quebec, CA) were implanted above the stratum oriens of CA1. Electrodes were custom made by soldering a tungsten wire (bare wire: $0.075 \mathrm{~mm}$, coating thickness quadruple PTFE: $0.018 \mathrm{~mm}, 99.98 \%$ purity, ADVENT Research Materials Itd., Oxford, EN) into a gold pin (Conrad, DE). Prior to implantation, electrodes were glued to the optic cannulas using a drop of dental cement (Cyano Veneer Pulver A2) mixed to the special 
glue (Cyano Veneer, Fast-retarder, Hager \& Werken GmbH \& Co. KG, Duisburg, DE). Electrodes were cut $250 \mu \mathrm{m}$ longer than the optic cannulas to target the stratum radiatum of CA1. This ferrule-electrode arrangement was stereotactically implanted at the same AP and ML coordinates as the injection sites but at a depth of $900 \mu \mathrm{m}$ from brain surface (from the optic fiber's tips). Reference and ground electrodes were additionally implanted in the cerebellum through small holes drilled into the cerebellar skull bone. Light guides and electrodes were fixed to the skull using a mixture of dental cement (Cyano Veneer Pulver A2) and special glue (Cyano Veneer, Fast-retarder, Hager \& Werken GmbH \& Co. KG, Duisburg, $\mathrm{DE})$. Mice were allowed at least one week of post-surgical recovery before experimental procedures began.

\section{Electrophysiological recordings in brain slices}

\section{Slice preparation}

Hippocampal coronal slices (300 $\mu \mathrm{m}$ thick) from wild type PV-Cre::APP/PS1 mice expressing rAAV2-Ef1a-DIO C1V1 (t/t)- TS-mCherry in the hippocampus were prepared with a Leica VT$1200 S$ vibratome (Leica Microsystems, Wetzlar, Germany) in ice-cold sucrose solution containing (mM): $60 \mathrm{NaCl}, 100$ sucrose, $2.5 \mathrm{KCl}, 1.25 \mathrm{NaH}_{2} \mathrm{PO}_{4}, 26 \mathrm{NaHCO}_{3}, 1 \mathrm{CaCl}_{2}, 5$ $\mathrm{MgCl}_{2}, 20$ glucose, oxygenated with $95 \% \mathrm{O}_{2}$ and $5 \% \mathrm{CO}_{2}$. After recovery for at least $30 \mathrm{~min}$ at $35{ }^{\circ} \mathrm{C}$ slices were transferred into standard ACSF with the following composition (mM): $125 \mathrm{NaCl}, 3 \mathrm{KCl}, 1.25 \mathrm{NaH}_{2} \mathrm{PO}_{4}, 26 \mathrm{NaHCO}_{3}, 2.6 \mathrm{CaCl}_{2}, 1.3 \mathrm{MgCl}_{2}, 15$ glucose) at room temperature.

Electrophysiological recordings with optogenetic stimulation

Electrophysiological recordings were obtained from neurons visualized by infrared DIC and fluorescence microscopy for mCherry identification (SliceScope with BX-RFA, Scientifica, East 25 Sussex, UK, Olympus, Hamburg, Germany). After confirmation of mCherry positive neuron in hippocampal CA1 area patch-clamp recordings were performed. Whole cell patch clamp recordings of either $\mathrm{PV}+$ interneurons or pyramidal neurons in hippocampus were performed using a ELC-03XS amplifier (npi electronic, Tamm, Germany) and digitalized at 50 $\mathrm{kHz}$ sampling rates using an ITC-18 interface board (HEKA) controlled by self-made acquisition software based on Igor Pro 6.3 (WaveMetrics, Portland, USA). $\mathrm{PV}^{+}$interneurons and pyramidal neurons were identified by mCherry expression and by the morphology of the cells, respectively. The cell types were also confirmed by the firing properties afterwards. The recording pipettes with a resistance of 4-6 $\mathrm{M} \Omega$ were filled with standard intracellular solution containing (mM): $140 \mathrm{~K}$-gluconate, $7 \mathrm{KCl}, 5 \mathrm{HEPES}$-acid, $0.5 \mathrm{MgCl}_{2}, 5$ phosphocreatine, 0.16 EGTA. All recordings were performed at $34{ }^{\circ} \mathrm{C}$ without correction for liquid junction potentials.Immediately after establishing the whole-cell configuration, the resting membrane potential (RMP) was measured. The membrane potential was adjusted to $-60 \mathrm{mV}$ by continuous current injection and a set of 500 ms hyper- and depolarizing stimuli (from -200 to $+500 \mathrm{pA}$ ) was applied. Optogenetic stimulation was performed with a $561 \mathrm{~nm}$ continuous wave solid-state laser (OBIS 56180 LS FP, Coherent, Santa Clara, USA) coupled to a light fiber. The light fiber tip was placed in a distance of $\leq 5 \mathrm{~mm}$ to the slice. Cells were stimulated with $8.5 \mathrm{~Hz}$ ( $15 \mathrm{~ms}$ pulse width) either without or with $\leq 30 \mathrm{~ms}$ random jitter of light for $5 \mathrm{~s}$

\section{Immunohistochemistry}

At the end of the experimental procedures, mice were transcardially perfused using 1xPBS and their brains were fixed overnight in $4 \%$ paraformaldehyde (PFA, Carl Roth $\mathrm{GmbH}+\mathrm{KG}$, Karlsruhe, DE). Afterwards, coronal slices were cut using a vibratome (Leica VT 1200S, Leica Byosystems Nussloch $\mathrm{GmbH}$, Heidelberg, DE) at a thickness of $100 \mu \mathrm{m}$. $\mathrm{PV}^{+}$ 
interneurons were stained using an $\alpha-\mathrm{PV}^{+}$primary antibody $(1: 1000, \mathrm{PV}-25$, Swant, Marly, $\mathrm{CH}$ ) diluted in the permeabilizing solution: $2 \%$ bovine serum albumin (2\% BSA, Carl Roth $\mathrm{GmbH}+$ Co. KG, Karlsruhe, DE), 2\% Triton X100 in 1xPBS (AppliChem GmbH, Darmstadt, $\mathrm{DE})$ and $10 \%$ normal goat serum in $1 \mathrm{x}$ PBS $(10 \%$ NGS, Thermo Fisher Scientific, Darmstadt, $\mathrm{DE})$. Slices were incubated overnight with the primary antibody solution at room temperature on a shaker $(300 \mathrm{rpm})$. On the next day, the solution was removed and the slices were washed in 1xPBS. A rabbit anti-mouse-Alexa488 antibody, diluted in $3 \%$ BSA/PBS, was used as secondary antibody $(1: 400$, rabbit $\alpha$-mouse, Invitrogen, Darmstadt, DE) for 2.5 hours at room temperature (300 rpm). Upon removal of the secondary antibody solution, slices were washed with $1 \%$ TritonX100 in 1x PBS for 10 minutes and finally with 1x PBS. Slices were mounted using fluorescent mounting medium (Dako, Deutschland $\mathrm{GmbH}$, Hamburg, DE). Confocal microscopy pictures were acquired using a LSM700 system (Carl Zeiss Microimaging $\mathrm{GmbH}$, Jena, $\mathrm{DE}$ ).

\section{Behavioral procedures}

\section{LFP recordings in the open field}

LFPs were recorded while mice explored a squared open field $(50 \times 50 \mathrm{~cm})$. Mice were let freely move in the arena for a maximum of 60 minutes and were videotaped for offline analyses. LFPs were sampled during the whole session. Mice were recorded until a minimum of 2 sweeps reaching a threshold activity criterion was reached (total travelled distance $>100 \mathrm{~cm}$ ). A custom-made faraday cage and a white curtain surrounded the open field, and no specific cues were added. The arena was wiped with $70 \%$ ethanol and water after every mouse.

\section{Optogenetic stimulation in the open field}

These experiments were performed in the same open field arena, room, and with the same conditions as mentioned above (open field). To verify that light stimulation of PV+ interneurons could drive theta frequencies in vivo, we stimulated these interneurons at 5,8 , 10 and $15 \mathrm{~Hz}$ with a $561 \mathrm{~nm}$ wavelength laser (OBIS $561 \mathrm{LS}$ - laser system, Coherent, Santa Clara, USA) while the mice freely explored the environment. Laser power and pulse duration were kept constant at $20 \mathrm{~mW}$ and $15 \mathrm{~ms}$, respectively. Power at the end of each optic fiber was $9 \pm 1 \mathrm{~mW}$. Light was bilaterally delivered to the brain using a rotating connector that served as a laser beam splitter. Light was equally distributed into two fiber optic patch cords (P54997-04, CM3-SMC, core: 300 um, NA:022, Doric lenses, Quebec, $\mathrm{CA}$ ), which were in turn connected to the optic fiber adaptors on the intracranially implanted ferrule. After every mouse, the arena was wiped with $70 \%$ ethanol and water.

\section{Novel object recognition}

Mice performed a novel object recognition (NOR) test in a squared arena $(50 \times 50 \mathrm{~cm})$ as described above (open field). During the sample phase, mice explored the arena, which contained two identical objects (two flasks filled with brown soil) placed in the center. The sample phase consisted of 3 exposures to the arena ( 6 minutes each), interspaced by 15 minutes. After 24 hours, one of the two familiar objects was replaced by a novel one (a metallic cylinder), and the mice were placed back in the arena for 10 minutes. The site of entrance into the arena and the position of the novel object were pseudo-randomized between groups. During both sample and test phases, LFPs were recorded and a feedback optogenetic stimulation protocol was applied (see stimulation protocols). LFPs were acquired 
from only one of the two recording electrodes. After every mouse, the arena and the objects were wiped with $70 \%$ ethanol and water.

\section{LFP recordings and stimulation protocols}

Setup

All electrodes were connected by means of custom assembled $2 \mathrm{~m}$ long insulated cable (single conductor wire LifY, $0.05 \mathrm{~mm}^{2}$ and $0.8 \mathrm{~mm}$ insulation, Conrad, DE) ending in a pinsocket on one end and connected to the headstage on the other. LFPs were sampled at 25 or $10 \mathrm{kHz}$ using an amplifier system and two portable headstages (NPI electronic $\mathrm{GmbH}$, Tamm, DE). The signal was low-pass filtered at $700 \mathrm{~Hz}$ and digitalized with an ITC-18 interface board (HEKA, New York, USA). The IgorPro software v. 6.22A (Wavemetrics, Portland, USA) was used for data collection and control of the laser stimulation. LFP data was downsampled to $1 \mathrm{kHz}$ for offline analysis.

\section{Closed-loop stimulation protocol}

Rhythmic stimulation. Using a custom designed procedure in IgorPro, a closed-loop stimulation was established to modulate theta frequencies in the local field potential: In intervals of $500 \mathrm{~ms}$ a Fourier transform of the past 4 seconds of LFP recording was calculated using a fast Fourier transformation. A clear peak in the theta band $(4-12 \mathrm{~Hz})$ was defined as a ratio of the peak theta amplitude to the average theta amplitude exceeding 4 . Whenever this criterion was fulfilled, a stimulation interval at the detected peak frequency plus $0.5 \mathrm{~Hz}$ with $15 \mathrm{~ms}$ pulse duration was initiated and protracted for 5.5 seconds. After each stimulation event, no new stimulation was initiated for at least 5 seconds to avoid detection of the induced activity.

Arrhythmic stimulation. The protocol described above was modified with a random jitter that followed a uniform distribution in the interval $[-30 \mathrm{~ms},+30 \mathrm{~ms}]$ to shift the timing of light pulses. This caused the randomization of the pulses across the stimulation interval. Every stimulation interval produced a different randomized set of pulses. However, on average, the stimulation frequency was the detected theta frequency plus $0.5 \mathrm{~Hz}$ as during the rhythmic stimulation.

\section{Optogenetic stimulation in the open field}

Stimulation protocols were designed in IgorPro. A 20 second protocol was employed to test whether stimulation at $5,8,10$ or $15 \mathrm{~Hz}$ would increase the amplitude of the respective stimulation frequency in the LFP. The protocol consisted of 5 seconds of baseline followed by 5 seconds of stimulation and further 10 seconds of recording in absence of stimulation. Laser power $(20 \mathrm{~mW})$ and light pulses (15 ms) were kept constant.

\section{Data analysis}

LFP data was analyzed using custom-made MATLAB scripts (R2013B, Mathworks, Natick MA, USA) or with IgorPro v. 6.22A (Wavemetrics, Portland, USA). Only one of the two implanted electrodes was used for LFP recordings throughout all the behavioral experiments.

\section{Analysis of whole-cell recordings}

All whole cell patch clamp recordings were analyzed using lgor Pro 6.3. From first to tenth of IPSPs in pyramidal neurons were analyzed for IPSP peak amplitude and area. IPSPs area was calculated for $55 \mathrm{~ms}$ from onset of light stimulation. 


\section{Baseline recordings}

To detect differences in theta parameters between wild type and transgenic animals, values were extracted from spectrograms produced with the wavelet method in MATLAB, using the FieldTrip toolbox ${ }^{27}$. Theta parameters (peak theta frequency, amplitude and duration of theta phases) were calculated for each recording sweep (3 minute per sweep). Only sweeps where the mice were actively exploring were included in the analysis (total travelled distance $>100 \mathrm{~cm}$ ). Intervals of theta oscillations were defined as epochs when theta amplitude (5-12 $\mathrm{Hz}$ ) increased by a ratio of 6 (arbitrarily set) over the delta band $(4-5 \mathrm{~Hz})$. The peak theta frequency was first averaged over sweeps for every mouse (minimum 2 sweeps, maximum 6). Subsequently, these values were averaged for each genotype. Travelled distance, mean velocity and time spent in the center were automatically analyzed in EthoVision XT (Noldus, Wageningen, NL). Center of the arena was defined as the squared portion of the open field being $5 \mathrm{~cm}$ distant from every wall of the arena.

\section{Optogenetic stimulation in the open field}

Fourier transformations of baseline and stimulation intervals were computed with IgorPro. The peak amplitude of the frequency of interest $(5,8,10$ or $15 \mathrm{~Hz})$ was normalized to baseline intervals ( 5 seconds prior to stimulation), and fold increase in the peak amplitude of the frequency of interest over baseline during stimulation intervals was calculated. Four to six stimulation protocols were applied per mouse, and the fold increase of the frequency of interest over baseline was averaged first per mouse, then per treatment group.

\section{Novel object recognition}

Travelled distance, total exploration time, mean velocity and time spent at objects were measured in EthoVision XT (Noldus, Wageningen, NL). An area of ca. $3 \mathrm{~cm}$ was drawn around both familiar and novel objects, and nose point entry into that arena was considered as object exploration. The discrimination index (DI) was calculated as the percentage of time spent exploring the novel object over the total exploration time: (novel object exploration time / total exploration time $)^{\star} 100 \%$. The behavior of the mice was analyzed for 5 minutes.

\section{Statistical analyses}

Statistical tests were carried out with GraphPad Prism 7 (GraphPad Software Inc., California, USA). Two-tailed unpaired Student's t-tests were used to compare theta parameters (Fig. 1c and d; , Supplementary fig. 1a) and movement parameters (Figure 1e, f). A two-tailed MannWhitney test was used to compare not normally distributed theta power values (theta/delta ratio; Supplementary figure $1 \mathrm{~b}$ ). Two-tailed unpaired Student's t-tests were used to compare effect of light stimulation at each frequency $(5,8,10,15 \mathrm{~Hz})$ between Sham and C1V1 expressing mice (Figure $2 \mathrm{~d}$ and Supplementary figure 4d). Differences in discrimination indices during the NOR test (Figure $3 \mathrm{~g}$ ) and locomotion parameters (Supplementary figure 2b) were compared using a two-way ANOVA followed by Holm-Sidak's multiple comparison correction. Two-tailed unpaired Student's t-tests were used to compare DIs and travelled distances of mice stimulated with the arrhythmic feedback stimulation protocol (Fig. 3h and Supplementary figure 2d). A two-tailed unpaired Student's t-test was used to compare regression slopes (Supplementary figure $3 \mathrm{e}$ and $\mathrm{k}$ ). All data was tested for normal distribution using the D'Agostino \& Pearson normality test. When the number of values was too small to carry out a normal distribution test, normal distribution was assumed. 